\title{
BMJ Open Rationale and protocol for the After Diabetes Diagnosis REsearch Support System (ADDRESS): an incident and high risk type 1 diabetes UK cohort study
}

Helen C Walkey, ${ }^{1}$ Akaal Kaur, ${ }^{1}$ Vassiliki Bravis, ${ }^{1}$ Ian F Godsland, ${ }^{1}$ Shivani Misra, ${ }^{1}$ Alistair J K Williams, ${ }^{2}$ Polly J Bingley, ${ }^{2}$ David B Dunger, ${ }^{3}$ Nick Oliver, ${ }^{1}$ Desmond G Johnston ${ }^{1}$

To cite: Walkey HC, Kaur A, Bravis V, et al. Rationale and protocol for the After Diabetes Diagnosis REsearch Support System (ADDRESS): an incident and high risk type 1 diabetes UK cohort study. BMJ Open 2017;7:e013956. doi:10.1136/ bmjopen-2016-013956

- Prepublication history for this paper is available online. To view these files please visit the journal online (http://dx.doi. org/10.1136/bmjopen-2016013956).

Received 19 August 2016 Revised 24 April 2017 Accepted 6 June 2017

\section{(a) CrossMark}

${ }^{1}$ Department of Medicine, Imperial College London, London, UK

${ }^{2}$ School of Clinical Sciences, University of Bristol, Bristol, UK ${ }^{3}$ Department of Paediatrics, University of Cambridge, Cambridge, UK

Correspondence to

Dr. Helen C Walkey; h.walkey@ imperial.ac.uk

\section{ABSTRACT}

Introduction Type 1 diabetes is heterogeneous in its presentation and progression. Variations in clinical presentation between children and adults, and with ethnic group warrant further study in the UK to improve understanding of this heterogeneity. Early interventions to limit beta cell damage in type 1 diabetes are undergoing evaluation, but recruitment is challenging. The protocol presented describes recruitment of people with clinicianassigned, new-onset type 1 diabetes to understand the variation in their manner of clinical presentation, to facilitate recruitment into intervention studies and to create an open-access resource of data and biological samples for future type 1 diabetes research.

Methods and analysis Using the National Institute for Health Research Clinical Research Network, patients $>5$ years of age diagnosed clinically with type 1 diabetes (and their siblings) are recruited within 6 months of diagnosis. Participants agree to have their clinical, laboratory and demographic data stored on a secure database, for their clinical progress to be monitored using information held by NHS Digital, and to be contacted about additional research, in particular immunotherapy and other interventions. An optional blood sample is taken for islet autoantibody measurement and storage of blood and DNA for future analyses. Data will be analysed statistically to describe the presentation of incident type 1 diabetes in a contemporary UK population.

Ethics and dissemination Ethical approval was obtained from the independent NHS Research Ethics Service.

Results will be presented at national and international meetings and submitted for publication to peer-reviewed journals.

\section{INTRODUCTION}

Type 1 diabetes (T1D) is the most common form of diabetes in childhood, but it is frequently diagnosed in adults. ${ }^{1}$ In the UK it is most common in people of white European descent, but also affects other ethnic groups, ${ }^{2}$ and it is heterogeneous in initial
Strengths and limitations of this study

- Children and adults (including older adults) are characterised soon after disease onset.

- There is wide geographical spread of ascertainment.

- The study allows differences in presentation of type 1 diabetes to be assessed by ethnic group.

- There is no attempt to capture information on all incident cases.

- Assessment of C-peptide and genetic risk markers is desirable in the characterisation of new-onset type 1 diabetes, but beyond the scope of this protocol. However, the After Diabetes Diagnosis REsearch Support System resource will enable precisely such future studies.

clinical presentation and in its progression. Markers of autoimmunity, such as antibodies to islet antigens, most commonly glutamate decarboxylase (GADA), insulin itself, islet antigen 2 (IA-2A) and zinc transporter-T8 (ZnT-8A), are frequently, but not always detectable at onset in people with a clinical diagnosis of T1D. ${ }^{4-6}$ Measurement of autoantibodies is advocated by many to aid diabetes classification, ${ }^{3-9}$ but only recommended in the UK when knowledge of autoantibody status (positive/negative) would have implications for clinical management or access to treatment (eg, 'insulin pump' therapy). ${ }^{10}$ Few T1D cohorts include both children and adults and, where studied, clinical characteristics and autoantibody frequencies have been found to vary between children and adults at onset. ${ }^{11-15}$ Additionally, descriptions of adult-onset diabetes with autoantibodies have highlighted differences in T1D phenotype at onset between adults and children. ${ }^{1617}$ Reports of the variation of autoantibody status 
and frequency with ethnicity are also scarce and have revealed a somewhat conflicting picture. ${ }^{618-20}$ Differences in presenting features of T1D with autoantibody status warrant further investigation in children and adults in the multiethnic UK population to improve understanding of the heterogeneity of T1D. People with T1D have a lifelong dependency on treatment with exogenous insulin, resulting from an autoimmune destruction of pancreatic beta cells. Early intervention therapies are emerging that aim to limit this autoimmune destruction and preserve beta cell function. ${ }^{21}{ }^{22}$ Preservation of even modest levels of insulin secretion has been shown to reduce the risk of developing diabetic complications, improve glycaemic control and may also protect against severe hypoglycaemia. ${ }^{2324}$ Many investigational therapies target new-onset T1D - at a stage when there is still significant insulin secretion to preserve. Recruitment to trials in new-onset T1D is challenging, in part because individual centres see a relatively small number of incident cases per year. The aims of the After Diabetes Diagnosis REsearch Support System (ADDRESS) are therefore the following:

1. to characterise people with clinician-assigned, newonset T1D, demographically, clinically and by islet autoantibody status, in a national, multiethnic cohort, and to perform hypothesis-generating analyses investigating the heterogeneity of clinical presentation

2. to use the cohort of children and adults with incident T1D and their siblings to support recruitment into T1D trials and other clinical research studies by providing them with information about studies for which they might be eligible (initially in new-onset T1D, and with time, studies in established T1D, or studies for firstdegree relatives), thereby also increasing awareness of opportunities to participate in research among patients and their families

3. to establish an open-access resource of data and biological samples, including DNA, collected close to diagnosis for use in other T1D research, forming, in particular, a foundation for prospective studies from the time of diagnosis.

There are a number of ongoing initiatives in the USA and Europe to characterise people with incident T1D, some of which include the banking of biological samples and open access to samples or data, some that include the study of first-degree relatives, and some that also support the conduct of clinical trials. Exemplar initiatives are summarised in table 1, in comparison with ADDRESS. There are T1D registries in the UK primarily set up to drive improvements in clinical care, and the notable open-access Warren repository, ${ }^{25}$ established to further understanding of T1D genetic susceptibility. There are, however, no national, multiethnic collections of data and biological samples from both children and adults with incident T1D in the UK. The Scottish Health Research register SHARE is an example of another national resource that supports recruitment to clinical research, although unlike ADDRESS it does not focus on new-onset
T1D. ${ }^{26}$ The features of these UK-specific T1D registries and resources are summarised in table 2.

In 2006 the National Institute for Health Research Clinical Research Network (NIHR CRN) was set up as the research delivery arm of the National Health Service (NHS) in England. The universal health coverage of the NHS, coupled with the NIHR CRN, makes the UK a particularly suitable environment for studies in new-onset T1D. This is further strengthened via linkage with the ADDRESS new-onset T1D ascertainment network, which is dedicated to identifying people with incident T1D shortly after diagnosis and offering them and their siblings entry into T1D clinical research studies. ADDRESS is a partner in the T1DUK Consortium, formed in 2015 to promote, support and develop immunotherapy research in the UK via a network of centres to conduct clinical trials, and a mechanistic core to conduct state-of-the-art assays to study the immunology of T1D and the mechanisms of action of immunotherapies.

\section{METHODS AND ANALYSIS}

\section{Establishment of the cohort}

The After Diabetes Diagnosis REsearch Support System (previously referred to as ADDRESS or ADDRESS-2, hereafter as ADDRESS) started in pilot form in 2008 to establish the 'proof of concept' that ascertainment and recruitment of people with incident T1D shortly after diagnosis were feasible in substantial numbers using the resources of the NIHR CRN. Patients with a clinician-assigned diagnosis of T1D were recruited if they agreed to have their data on a database and to be contacted about other research projects for which they might be eligible. Clinical and demographic information, including the participant's unique NHS identifier, was obtained for analysis, but no biological samples were collected. This pilot was established in 78 recruiting hospitals in England and Wales. NIHR CRN staff recruited 645 participants between 2008 and 2010. With cofunding from Diabetes UK and the Juvenile Diabetes Research Foundation, and with separate NHS Research Ethics Committee review, the project was expanded to its present and ongoing form in January 2011 in which 156 hospitals are participating in 124 NHS Trusts across England and Health Boards in Wales. Figure 1 illustrates the timeline of development of ADDRESS, and figure 2 shows the locations of centres participating currently. The NIHR CRN structure provides research support via 15 local branches in England and a related system exists to support diabetes research in Wales.

\section{Inclusion and exclusion criteria}

During the first 3 years of the current study (2011-2014), people with a clinician-assigned diagnosis of T1D aged 5-60 years were recruited within 6 months of diagnosis. From October 2014 the upper age limit was removed to allow newly diagnosed adults over the age of 60 to participate. People initially diagnosed with another diabetes 


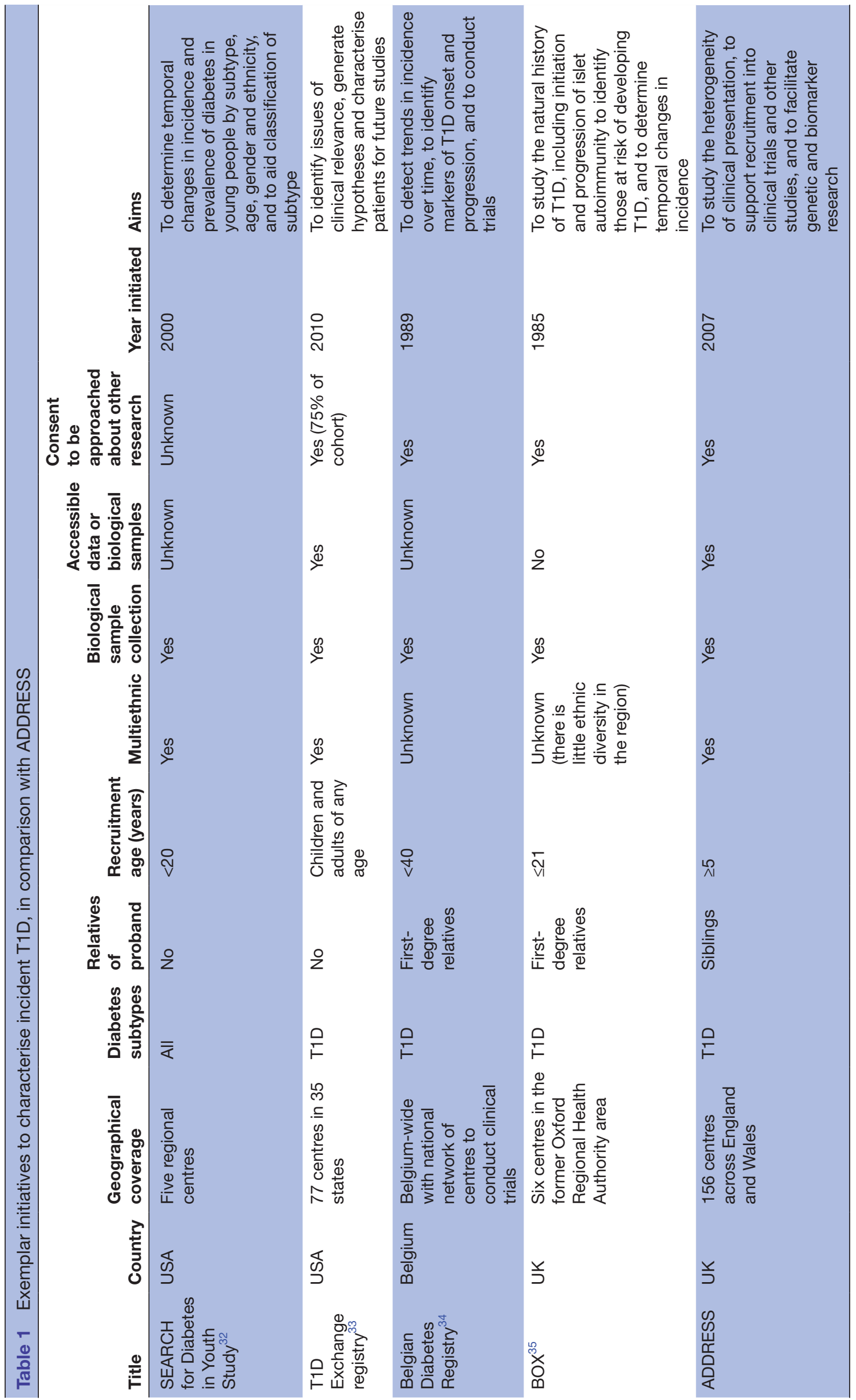

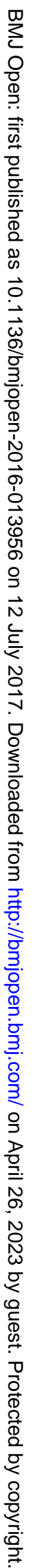




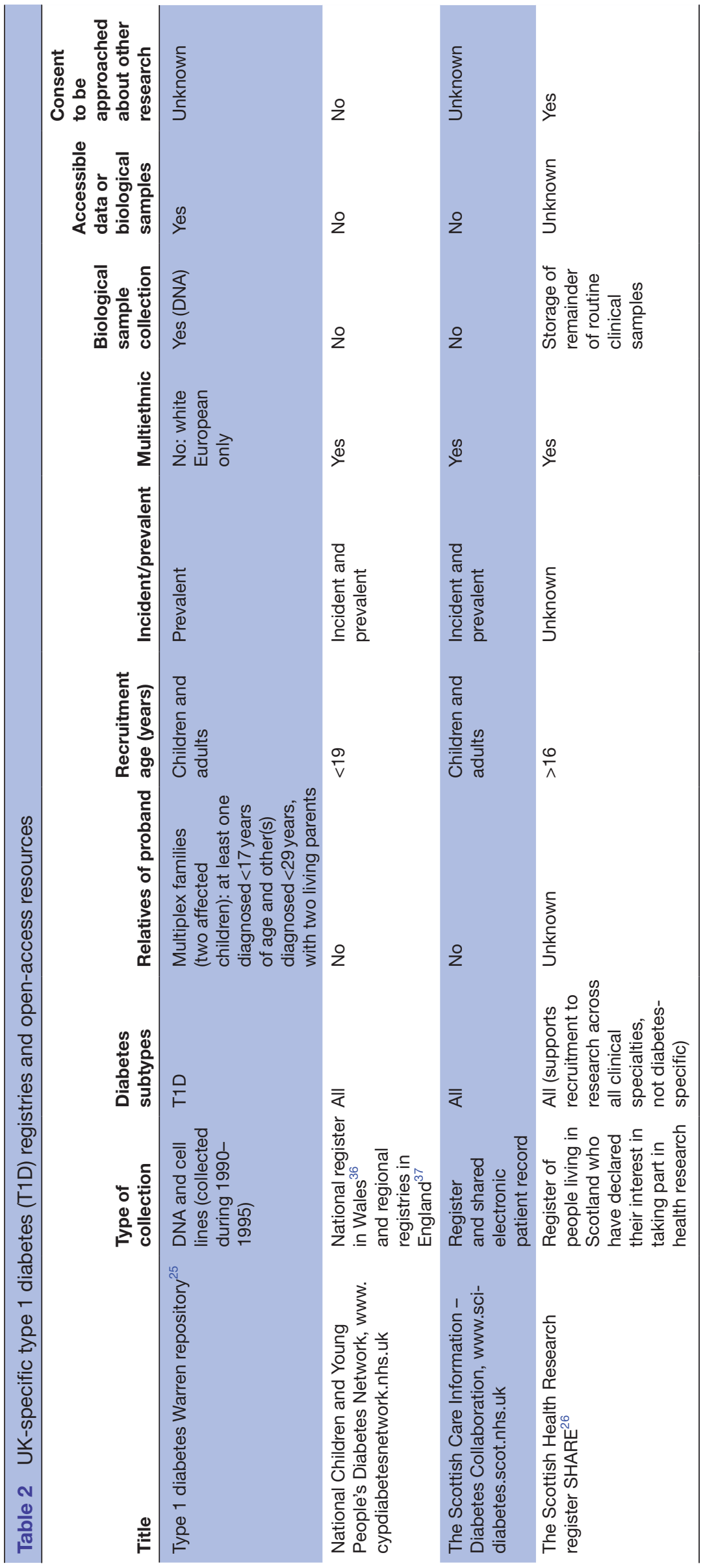

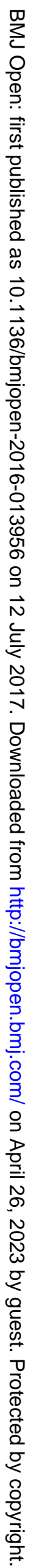




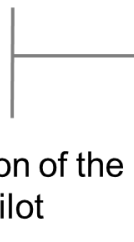

\section{|}
Initiation of the
pilot
Expansion to measure islet
autoantibodies, collect DNA
and blood samples, and
recruit unaffected siblings

\section{Ongoing recruitment}

Figure 1 Study timeline.

subtype and later reassigned a diagnosis of T1D are eligible if still within 6 months of the initial diagnosis. Adults who are not competent to give consent are excluded. People who took part in the pilot form of ADDRESS are eligible to be recruited to the current study, and their individual data are transferred from the pilot into the current study.

\section{Sample size}

The annual incidence of T1D in the UK is 22-30 per 100000 in the $0-14$ years age group, with an estimated $76 \%$ of incident cases aged $5-14$ years $^{27}$. There is a paucity of incidence data for adults worldwide, but one UK study reported an incidence of 12 per 100000 for those aged $15-34$ years. ${ }^{28}$ Therefore, we estimate that $3900-4500$ children and adults will be diagnosed per year in the current recruitment age range in England and Wales.

\section{Recruitment}

Methods vary depending on local resources and preferences, but local researchers (NIHR CRN research nurses and/or other local research nurses and physicians) perform the recruitment mainly via secondary care and specialist diabetes centres, as patients (both children and adults) are mostly seen clinically in these settings. Recruiting staff in the NIHR CRN have been trained to approach patients and families with sensitivity soon after diagnosis and to take informed consent. Permission is sought from the patient or parent/guardian to approach siblings. The patients, their siblings and the parent/ guardian for children aged 5-15 years are provided with age-specific information sheets about the study by the local researcher. Informed consent is sought at least 1 week after diagnosis and at least 48 hours after the information sheets have been given. Recruitment support initiatives have included providing NHS clinical care teams with information about T1D research, displaying posters about ADDRESS and providing patients with leaflets introducing clinical research and ADDRESS in clinic and in packs for newly diagnosed patients. A website provides additional information.

\section{Consent}

ADDRESS operates a two-tier consent model: for tier 1, participants provide written informed consent for their health and contact details to be held on a database and for their being contacted about studies. They also consent to follow-up using information held by NHS Digital and the Office for National Statistics (ONS). With additional second-tier consent, participants may choose to donate blood samples for islet autoantibody analysis and storage of blood and DNA for future use in diabetes research.

\section{Data collection}

At the time of recruitment participant information is collected via interview with the participant or parent/ guardian, and from specialist care medical records, as shown in table 3. Ethnicity is self-reported as one of 16 categories, following UK ONS guidance on collecting ethnic group data. A diagnosis of parental or grandparental diabetes is recorded without subtype because historical classifications may be incorrect or incomplete. ${ }^{29}$ Laboratory test results are recorded from those tests performed locally as part of routine clinical care. A diagnosis of ketoacidosis at presentation is recorded if assigned by the local clinical team, or if all of the following were documented in the clinical records: glucose $>11 \mathrm{mmol} / \mathrm{L}$; blood ketone bodies $>3 \mathrm{mmol} / \mathrm{L}$ or urine ketones levels positive ++; $\mathrm{pH}<7.3$; and/or bicarbonate $<15 \mathrm{mmol} / \mathrm{L}$. Except for the additional specialist care information available for patients, similar data are collected at baseline for siblings.

\section{Project-specific blood sampling}

Sample donation is a voluntary component of ADDRESS, currently for participants with T1D only (prior to October 2014, siblings were also given the option of donating samples). Random blood samples are collected and sent at ambient temperature via standard post to the Public Health England European Collection of Cell Cultures at Porton Down, UK, which operates in accordance with the UK Human Tissue Act 2004. Serum (from a serum separation tube) and peripheral blood lymphocytes (PBLs, from an acid citrate dextrose tube) are separated on arrival. An EDTA tube is frozen for later DNA extraction (median: 2 (IQR: 1-3) days after sampling). The samples are stored in coded, pseudoanonymised format and include serum from coagulated blood, extracted and 


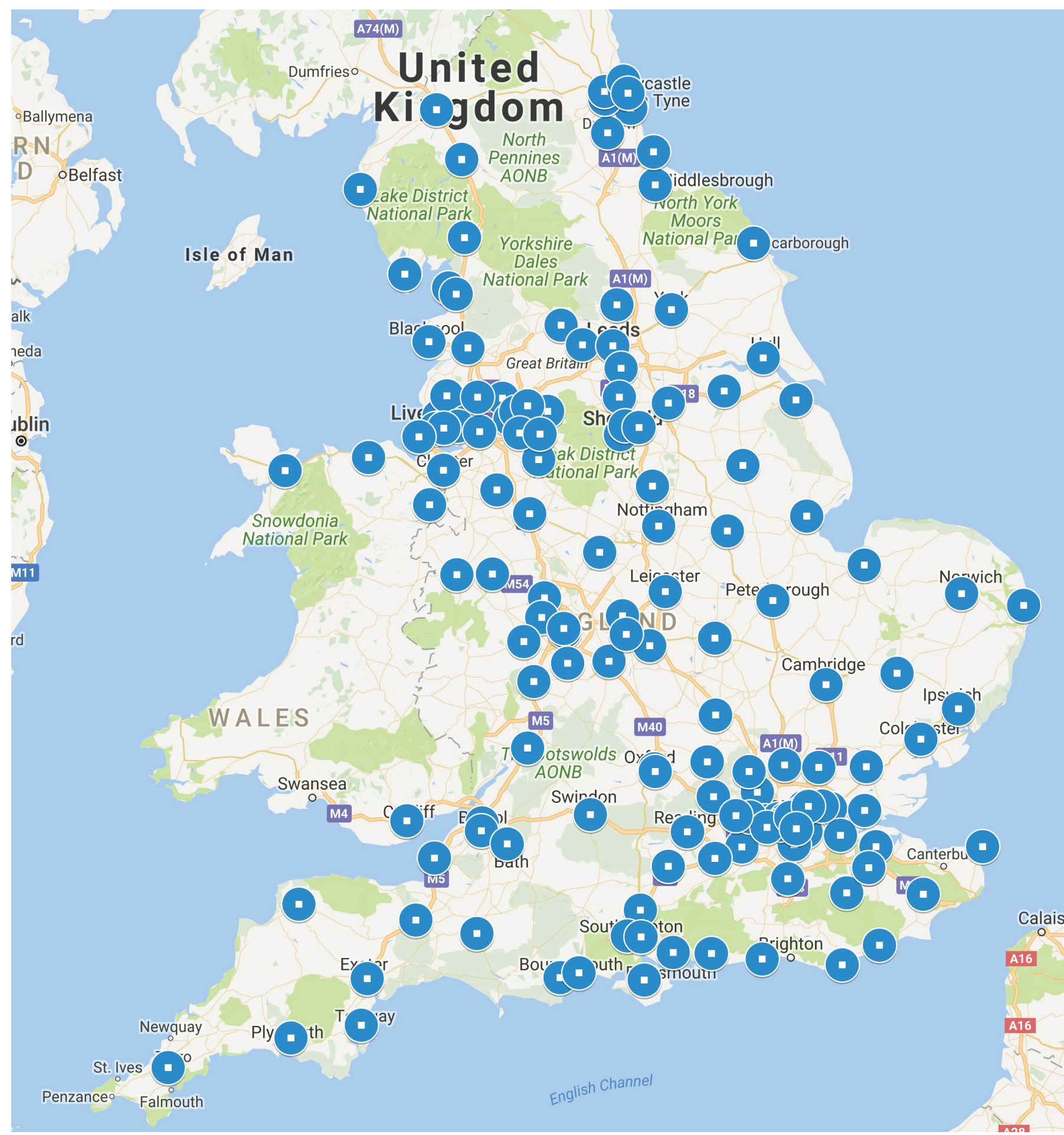

Figure 2 Map with circles showing the locations of the 156 recruiting centres in 124 English NHS Trusts and Welsh Health Boards (an estimated $84 \%$ of the total number that provide acute care services). Numbers of centres recruiting from both paediatric and adult clinics, the paediatric clinic only, and the adult clinic only are 80, 32 and 44, respectively.

resuspended DNA, PBLs, and lymphoblastoid cell lines (LCLs) derived by Epstein-Barr virus transformation of thawed PBLs. Samples are stored at $-80^{\circ} \mathrm{C}$ and PBLs and LCLs are placed in liquid nitrogen for cryopreservation for the duration of the study. An aliquot $(0.5 \mathrm{~mL})$ of serum from each blood sample is sent to the University of Bristol for autoantibody measurements.
Islet autoantibody measurement

Autoantibodies to GADA, IA-2A and ZnT-8A are measured in participants with T1D using established radiobinding assays. ${ }^{30}$ Insulin autoantibodies are not measured as these people are receiving exogenous insulin therapy, which could itself induce an immune response. 
Table 3 Data and associated methods of collection for participants with incident T1D and for sibling participants at the time of recruitment

\begin{tabular}{|c|c|c|c|}
\hline Data & Incident T1D & & Sibling \\
\hline & $\begin{array}{l}\text { Via interview } \\
\text { or at visit }\end{array}$ & $\begin{array}{l}\text { Via or validated } \\
\text { against medical } \\
\text { records }\end{array}$ & $\begin{array}{l}\text { Via interview } \\
\text { or at visit }\end{array}$ \\
\hline Contact details and unique NHS identifier & $\mathrm{X}$ & & $\mathrm{X}$ \\
\hline $\begin{array}{l}\text { General practitioner (GP) details (GP is informed of the person's } \\
\text { participation) }\end{array}$ & $\mathrm{X}$ & & $\mathrm{X}$ \\
\hline Demographic information including self-reported ethnic origin & $\mathrm{X}$ & & $\mathrm{X}$ \\
\hline $\begin{array}{l}\text { Date of diagnosis, clinical presentation and duration of symptoms } \\
\text { (presentation with diabetic ketoacidosis, polyuria/polydipsia, weight loss, } \\
\text { fatigue, abdominal pain, }{ }^{*} \text { fasting or random plasma glucose) }\end{array}$ & $\mathrm{X}$ & $\mathrm{x}$ & \\
\hline $\begin{array}{l}\text { Family medical history including parental and grandparental history of } \\
\text { diabetes, hypertension, myocardial infarction and stroke, along with sibling } \\
\text { demographics and diabetes history }\end{array}$ & $\mathrm{x}$ & & $\mathrm{X}$ \\
\hline $\begin{array}{l}\text { Clinical measures, including blood pressure, weight and height using } \\
\text { standard protocols }\end{array}$ & $\mathrm{X}$ & & $\mathrm{X}$ \\
\hline $\begin{array}{l}\text { Blood biochemistry including glycated haemoglobin }(\mathrm{HbA} 1 \mathrm{c})(\mathrm{mmol} / \\
\mathrm{mol}) \text {, fasting or random plasma glucose }(\mathrm{mmol} / \mathrm{L}) \text {, oral glucose tolerance } \\
\text { test results (mmol/L) if performed locally and C-peptide levels ( } \mathrm{nmol} / \mathrm{L}) \text { if } \\
\text { performed locally }\end{array}$ & & $\mathrm{x}$ & \\
\hline
\end{tabular}

${ }^{*}$ Collected for participants recruited from February 2015 onwards.

T1D, type 1 diabetes; NHS, National Health Service.

Antigens radiolabelled with ${ }^{35} \mathrm{~S}$ methionine are expressed using a TNT in vitro reticulocyte lysate quick coupled transcription/translation system kit (Promega, Madison, Wisconsin, USA) with plasmids encoding fulllength GAD65, the intracytoplasmic region of IA-2 (aa 606-979), and the 325-arginine (ZnT8R) and 325-tryptophan (ZnT8W) isoforms of the C-terminal region of ZnT8 (aa268-379). Immunocomplexes formed following incubation of sera with radiolabelled antigens are precipitated with Protein A-Sepharose (GE Healthcare Life Sciences, Little Chalfont, Bucks, UK), washed and counted in a TopCount beta counter (PerkinElmer, Waltham, Massachusetts, USA).

Results are expressed in digestive and kidney (DK) units/mL (GADA and IA-2A) or arbitrary units (ZnT8A) after reference to standard curves consisting of dilutions of patient sera in antibody-negative sera from healthy donors. Thresholds are set at the 97 th percentile of 974 control samples for GADA, the 98th percentile of 500 control samples for IA-2A and the 97.5th percentile of 523 healthy schoolchildren for ZnT8A. The GADA assay achieves a sensitivity of $74 \%$ at $96.7 \%$ specificity, while the
IA-2A, ZnT8RA and ZnT8WA assays achieve sensitivities of $72 \%, 60 \%$ and $46 \%$, respectively, at $100 \%$ specificity, in the 2015 Islet Autoantibody Standardization Program Workshop. Interassay coefficients of variation of high and moderate/low positive samples, respectively, were $18 \%$ and $18 \%$ for GADA, $19 \%$ and $17 \%$ for IA-2A, $22 \%$ and $16 \%$ for ZnT8RA, and $25 \%$ and $20 \%$ for ZnT8WA.

\section{Follow-up data collection and verification}

Participants with T1D are reviewed via their medical records at 4-8 months following diagnosis to verify that their original clinical diabetes classification has not been changed by their clinicians. Data reviewed at the follow-up are shown in table 4 .

An application to NHS Digital and the UK ONS is planned for tracing change of postal address, mortality flagging and access to hospital admission data (Hospital Episode Statistics).

\section{Database}

Data are entered into a secure electronic data capture and management system designed specifically for clinical 
Table 4 Data collection for participants with incident T1D at 4-8 months postdiagnosis and at least 2 months after the time of recruitment

\begin{tabular}{lc}
\hline Data & \multicolumn{1}{c}{ Incident T1D } \\
\hline & $\begin{array}{l}\text { Via medical } \\
\text { records }\end{array}$ \\
\hline $\begin{array}{l}\text { Confirmation of, or change in classification } \\
\text { of, diabetes subtype }\end{array}$ & $\mathrm{X}$ \\
$\begin{array}{l}\text { Occurrence of diabetic ketoacidosis since } \\
\text { diagnosis }\end{array}$ & $\mathrm{X}$ \\
$\begin{array}{l}\text { Any change in diabetes treatment or dose, } \\
\text { and concurrent medication }\end{array}$ & $\mathrm{X}$ \\
$\begin{array}{l}\text { Clinical measures, including blood pressure, } \\
\text { weight and height }\end{array}$ & $\mathrm{X}$ \\
$\begin{array}{l}\text { Blood biochemistry including glycated } \\
\text { haemoglobin (HbA1c) (mmol/mol), fasting } \\
\text { or random plasma glucose (mmol/L), oral } \\
\text { glucose tolerance test results (mmol/L) } \\
\text { if performed locally and C-peptide levels } \\
\text { (nmol/L) if performed locally }\end{array}$ & \\
$\begin{array}{l}\text { Details of diabetes structured education } \\
\text { offered/scheduled/completed* }\end{array}$ & $\mathrm{X}$ \\
\hline
\end{tabular}

All data are collected from medical records.

*Collected for participants recruited from February 2015 onwards. T1D, type 1 diabetes.

research. All information is stored in accordance with the UK Data Protection Act (1998).

\section{Recruitment into other studies}

Eligible participants are given information at intervals about specific diabetes trials/research studies by the central study team or local researchers. Information is retained on those previously contacted about research and whether or not they are currently participating in a study.

\section{Data analysis plan}

The collected data allow generation and testing of hypotheses about the presentation of clinician-assigned T1D and other characteristics early after diagnosis. Our aim is to compare characteristics between groups: for example, islet autoantibody positive and negative; ethnicity groupings of white European, South Asian (including Indian, Pakistani and Bangladeshi) and African-Caribbean (black African or black Caribbean); and children and adults. We aim also to investigate the relationships between insulin dose and glycaemic control. Recorded variables are categorised as 'Individual Characteristics' (eg, age, gender, child/adult, birth weight, ethnicity, history of other autoimmune disease, parent with diabetes, sibling with diabetes), 'Diabetes Presentation' (eg, ketoacidosis at T1D presentation, osmotic symptoms (polydipsia/ polyuria), weight loss, fatigue, symptom duration, initial insulin dose and autoantibody status) and 'Diabetes Characteristics' (eg, insulin dose, glycated haemoglobin (HbA1c) at time points after presentation).

\section{ETHICS AND DISSEMINATION}

Ethical approval was obtained from the NHS Research Ethics Committee - South Central-Berkshire (reference 10/H0505/85), and each participating NHS Trust submitted a Site-Specific Assessment in order to participate. The project is conducted in accordance with the recommendations for physicians involved in research on human subjects by the 18th World Medical Assembly, Helsinki 1964 and later revisions. The International Conference on Harmonisation Guideline for Good Clinical Practice (Topic E6 - 10 June 1996) and participant confidentiality are maintained throughout.

ADDRESS is an accessible resource for clinical and academic researchers, both as an infrastructure to identify and contact candidates for recruitment into T1D studies and as an open-access database and biological sample repository. Access is through a management committee comprised of lay people, scientists and clinical investigators representing relevant $\mathrm{T} 1 \mathrm{D}$ research interests. Researchers wishing to use the ADDRESS-2 resource can apply to the ADDRESS-2 Management Committee. The access process and associated documents are described on the study website (www.address2.org).

Ethical approval will be sought for continuing use of the data every 5 years (current approval is to the end of 2019). Every effort will be made to sustain this open-access resource beyond the present funding term. The database will be maintained by Imperial College London. The biological samples will continue to be stored for as long as there is funding to support the repository.

The results arising from this project will be presented at national and international meetings, and submitted for publication to peer-reviewed medical journals.

Acknowledgements The authors would like to thank Professor Nish Chaturvedi, now at University College London, and Dr Bob Goldstein at the Juvenile Diabetes Research Foundation for their input and advice.

Contributors The vision for an ascertainment network was that of DGJ in consultation with funders. Recruitment, data collection and analysis were designed and refined by DGJ, HCW, NO, VB, AK, SM and DBD. Islet autoantibody analysis was designed by PJB and AJKW. The statistical analysis plan was written by IFG. Procedures for open access were drafted by HCW and ratified by the ADDRESS-2 Management Committee.

Funding This work was supported by Diabetes UK grant number 09/0003919 and the Juvenile Diabetes Research Foundation grant number 9-2010-407. Recruitment is supported by staff at the National Institute for Health Research Clinical Research Network.

Competing interests None declared.

Ethics approval NHS Research Ethics Committee (South Central-Berkshire).

Provenance and peer review Not commissioned; externally peer reviewed.

Open Access This is an Open Access article distributed in accordance with the Creative Commons Attribution Non Commercial (CC BY-NC 4.0) license, which permits others to distribute, remix, adapt, build upon this work non-commercially, and license their derivative works on different terms, provided the original work is properly cited and the use is non-commercial. See: http://creativecommons.org/ licenses/by-nc/4.0/

(C) Article author(s) (or their employer(s) unless otherwise stated in the text of the article) 2017. All rights reserved. No commercial use is permitted unless otherwise expressly granted. 


\section{REFERENCES}

1. Diaz-Valencia PA, Bougnères $P$, Valleron AJ. Global epidemiology of type 1 diabetes in young adults and adults: a systematic review. BMC Public Health 2015;15:255.

2. Audit NPD. National Paediatric Diabetes Audit 2015-16 Report 1: care processes and outcomes: Royal College of Paediatrics and Child Health, 2017.

3. Chiang JL, Kirkman MS, Laffel LM, et al. Type 1 diabetes through the life span: a position statement of the American Diabetes Association. Diabetes Care 2014;37:2034-54.

4. Wenzlau JM, Juhl K, Yu L, et al. The cation efflux transporter ZnT8 (Slc30A8) is a Major autoantigen in human type 1 diabetes. Proc Natl Acad Sci U S A 2007;104:17040-5.

5. Hameed S, Ellard S, Woodhead HJ, et al. Persistently autoantibody negative (PAN) type 1 diabetes mellitus in children. Pediatr Diabetes 2011;12(3 Pt 1):142-9.

6. Perchard R, MacDonald D, Say J, et al. Islet autoantibody status in a multi-ethnic UK clinic cohort of children presenting with diabetes. Arch Dis Child 2015;100:348-52.

7. Hamman RF, Bell RA, Dabelea D, et al. The SEARCH for Diabetes in Youth study: rationale, findings, and future directions. Diabetes Care 2014;37:3336-44.

8. Tuomi T, Santoro N, Caprio S, et al. The many faces of diabetes: a disease with increasing heterogeneity. Lancet 2014;383:1084-94.

9. Leslie RD, Palmer J, Schloot NC, et al. Diabetes at the crossroads: relevance of disease classification to pathophysiology and treatment. Diabetologia 2016;59:13-20.

10. National Institute for Health and Care Excellence. Type 1 diabetes in adults: diagnosis and management: NICE Guideline, 2015.

11. Karjalainen J, Salmela P, Ilonen J, et al. A comparison of childhood and adult type I diabetes mellitus. N Engl J Med 1989;320:881-6.

12. Lohmann T, Sessler J, Verlohren HJ, et al. Distinct genetic and immunological features in patients with onset of IDDM before and after age 40. Diabetes Care 1997;20:524-9.

13. Vandewalle CL, Falorni A, Lernmark A, et al. Associations of GAD65and IA-2- autoantibodies with genetic risk markers in new-onset IDDM patients and their siblings. the Belgian Diabetes Registry. Diabetes Care 1997;20:1547-52.

14. Pozzilli P, Visalli N, Buzzetti R, et al. Metabolic and immune parameters at clinical onset of insulin-dependent diabetes: a population-based study. IMDIAB Study Group. immunotherapy Diabetes. Metabolism 1998;47:1205-10.

15. Sabbah E, Savola K, Ebeling T, et al. Genetic, autoimmune, and clinical characteristics of childhood- and adult-onset type 1 diabetes. Diabetes Care 2000;23:1326-32.

16. Hawa MI, Kolb H, Schloot N, et al. Adult-onset autoimmune diabetes in Europe is prevalent with a broad clinical phenotype: action LADA 7. Diabetes Care 2013;36:908-13.

17. Merger SR, Leslie RD, Boehm BO. The broad clinical phenotype of type 1 diabetes at presentation. Diabet Med 2013;30:170-8.

18. Wenzlau JM, Frisch LM, Hutton JC, et al. Changes in Zinc Transporter 8 Autoantibodies following type 1 Diabetes Onset: the type 1 Diabetes Genetics Consortium Autoantibody Workshop. Diabetes Care 2015;38(Suppl 2):S14-S20.

19. Stoupa A, Dorchy H. HLA-DQ genotypes - but not immune markers - differ by ethnicity in patients with childhood onset type 1 diabetes residing in Belgium. Pediatr Diabetes 2016;17:342-50.
20. Ong YH, Koh WCA, Ng ML, et al. Glutamic acid decarboxylase and islet antigen 2 antibody profiles in people with adult-onset diabetes mellitus: a comparison between mixed ethnic populations in Singapore and Germany. Diabet Med 2017.

21. Lernmark A, Larsson HE. Immune therapy in type 1 diabetes mellitus. Nat Rev Endocrinol 2013;9:92-103.

22. von Herrath M, Peakman M, Roep B. Progress in immune-based therapies for type 1 diabetes. Clin Exp Immunol 2013;172:186-202.

23. Steffes MW, Sibley $S$, Jackson $M$, et al. Beta-cell function and the development of diabetes-related complications in the diabetes control and complications trial. Diabetes Care 2003;26:832-6.

24. Kuhtreiber WM, Washer SL, Hsu E, et al. Low levels of C-peptide have clinical significance for established type 1 diabetes. Diabet Med 2015;32:1346-53.

25. Bain SC, Todd JA, Barnett AH. The British Diabetic Association-Warren Repository. Autoimmunity 1990;7:83-5.

26. McKinstry B, Sullivan FM, Vasishta S, et al. Cohort profile: the Scottish Research register SHARE. A register of people interested in research participation linked to NHS data sets. BMJ Open 2017; 7:e013351.

27. Patterson CC, Dahlquist GG, Gyürüs E, et al. Incidence trends for childhood type 1 diabetes in Europe during 1989-2003 and predicted new cases 2005-20: a multicentre prospective registration study. Lancet 2009;373:2027-33.

28. Imkampe AK, Gulliford MC. Trends in type 1 diabetes incidence in the UK in 0- to 14-year-olds and in 15- to 34-year-olds, 1991-2008. Diabet Med 2011;28:811-4.

29. Stone MA, Camosso-Stefinovic J, Wilkinson J, et al. Incorrect and incomplete coding and classification of diabetes: a systematic review. Diabet Med 2010;27:491-7.

30. Bonifacio E, Yu L, Williams AK, et al. Harmonization of glutamic acid decarboxylase and islet antigen-2 autoantibody assays for national institute of diabetes and digestive and kidney diseases consortia. $J$ Clin Endocrinol Metab 2010;95:3360-7.

31. Long AE, Gooneratne AT, Rokni S, et al. The role of autoantibodies to zinc transporter 8 in prediction of type 1 diabetes in relatives: lessons from the European Nicotinamide Diabetes intervention trial (ENDIT) cohort. J Clin Endocrinol Metab 2012;97:632-7.

32. SEARCH Study Group. SEARCH for Diabetes in Youth: a multicenter study of the prevalence, incidence and classification of diabetes mellitus in youth. Control Clin Trials 2004;25:458-71.

33. Beck RW, Tamborlane WV, Bergenstal RM, et al. The T1D Exchange clinic registry. J Clin Endocrinol Metab 2012;97:4383-9.

34. Gorus FK, Anselmo J, Keymeulen B. Type 1 (insulin-dependent) diabetes mellitus: an autoimmune, predictable and preventable disease? Lessons from national registries and new challenges to clinical biology. Acta Clin Belg 1993;48:96-108.

35. Bingley PJ, Gale EA. Incidence of insulin dependent diabetes in England: a study in the Oxford region, 1985-6. BMJ 1989;298:558-60.

36. O'Hagan M, Harvey JN, Brecon G; Brecon Group. Glycemic control in children with type 1 diabetes in Wales: influence of the pediatric diabetes specialist nurse. Diabetes Care 2010;33:1724-6.

37. Staines A, Bodansky HJ, Lilley HE, et al. The epidemiology of diabetes mellitus in the United Kingdom: the Yorkshire Regional Childhood Diabetes Register. Diabetologia 1993;36:1282-7. 